\title{
Une ethnographie réciproque
}

\section{Étudier les vies roms et non-roms en collaboration}

Paloma Gay y Blasco et Liria de la Cruz

Au printemps 1992, Liria de la Cruz était une jeune mère gitane qui vivait à Orcasitas, un quartier défavorisé du Sud de Madrid. Elle avait grandi dans les banlieues de la ville, où bidonvilles et HLM se côtoyaient, et où les Gitans se mêlaient aux plus pauvres des Payos ${ }^{1}$. Au côté de son mari, elle gagnait sa vie en vendant des vêtements sur les marchés. Pendant ce temps, Paloma Gay, une étudiante en anthropologie issue d'une famille de la classe moyenne d'origine payo, effectuait des recherches sur le terrain non loin de là, à Villaverde Alto, dans un ghetto gitan isolé, établi par le gouvernement, où résidaient de nombreux proches de Liria.

Paloma avait quitté Madrid pour poursuivre ses études à l'étranger et faisait son doctorat dans une université anglaise. Elle cherchait une famille gitane qui pourrait l'accueillir et Liria et son mari Ramón se sont proposés. Liria et Paloma sont rapidement devenues très proches.

Plus de vingt ans après, Liria ne vit plus avec Ramón et leurs enfants. En 2008, elle a rencontré un jeune Marocain, Younes Bziz, en est tombée amoureuse et a quitté sa famille pour débuter une nouvelle vie avec lui. La plupart des Gitans l'évitent, et Liria vit dans la précarité parmi les immigrants d'Afrique du Nord et d'Amérique Latine. Désormais maîtresse de conférence en anthropologie en Écosse, Paloma a décidé d'apporter son soutien à Liria, en l'aidant tout d'abord à s'enfuir, puis à exiger de voir sa plus jeune fille par le biais d'un tribunal payo. Cela signifiait trahir la confiance de la famille étendue de Liria, les Gitans à propos desquels elle avait écrit pendant de nombreuses années. À présent, aucune des deux

1 Pour les Gitans (Tsiganes espagnols/Roms), le mot Gitan possède des fortes connotations positives : il s'agit du seul mot qu'ils utilisent en référence à leur identité ethnique. Cependant, pour les Payos (non-Tsiganes/Roms), le terme Gitan a souvent un sens négatif. Bien que les Gitans utilisent le terme Payo pour tous ceux qui ne sont pas Gitans, quelle que soit leur origine nationale ou leur identité ethnique, ils font la distinction entre les Espagnols non-Gitans (Payos) et les immigrants de couleur (Marocains, Équatoriens, Péruviens, etc.) 
ne pouvait retourner dans le quartier de Madrid où Liria avait vécu et où Paloma avait débuté ses recherches.

La fuite de Liria nous a encore rapprochées et nous a amenées à faire le point sur notre relation. Au fil des jours, des semaines et des mois, nous avons pris des décisions qui ont changé nos vies et celles de la famille de Liria, et de Younes. Nous nous sommes également interrogées à propos de notre place dans le monde, de nous-mêmes et de l'anthropologie. Nous parlons ici de ce qui nous rassemble et ce qui nous éloigne en tant que Paya et Gitane, en tant que collaboratrices, amies, mères, épouses et travailleuses. Nous envisageons l'anthropologie comme ce qui nous a réunies.

Il ne s'agit pas là d'une biographie anthropologique. Paloma n'est pas uniquement l'anthropologue, l'interprète ou la traductrice des mots utilisés par Liria, de même que Liria n'est pas uniquement l'informatrice,

fournissant de la matière première, source d'explications et de discussions pour Paloma. À travers I'histoire de Liria, il faut également relater celle de Paloma, de son enfance, de son travail auprès des Gitans, ainsi que de sa décision d'aider Liria à s'enfuir. Nous évoquons toutes deux nos propres expériences et analysons la vie de l'autre pour nos lecteurs. L'accent est mis sur ce que nos deux histoires, entremêlées et parallèles, révèlent du point de vue de l'anthropologie, de l'amitié et de la femme espagnole, à la fois Paya et Gitane.

En réfléchissant ensemble à nos vies et à la manière dont nous nous sommes influencées au fil des ans, nous remettons en cause des divisions fondamentales de l'anthropologie, entre le terrain et le bureau, entre ceux et celles qui écrivent et ceux et celles qui sont le sujet, ceux et celles qui font l'apprentissage et ceux et celles au sujet desquels on apprend. Nous prenons également en compte d'autres divisions, entre femmes et hommes, Gitans et Payos, les gens pour qui subsister au quotidien dans l'Espagne du 21 ème siècle est plus facile et les gens pour qui cela s'avère plus difficile. Telles sont les divisions qui ont façonné nos vies et sont à la base même de notre amitié.

Ici, nous parlons de nous, et des gens qui nous entourent, mélange de souvenirs, d'histoires et d'ethnographie. Les thèmes que nous abordons sont importants pour nous et nous estimons qu'ils le sont également pour l'anthropologie. En racontant nos histoires, nous parlons d'appartenance et d'exclusion, de conformité et de transgression, d'oppression et de 
résistance dans les relations entre et parmi les Gitans, les Payos et les immigrants. En comparant nos familles, nos milieux d'origine, et nos parcours, nous parlons des inégalités qui façonnent l'Espagne contemporaine. Nous examinons notre relation et entreprenons une ethnographie auto/biographique réciproque, et de ce fait, nous envisageons une méthode, une raison d'être et un avenir pour l'anthropologie. Afin que nos deux voix soient visibles, nous utilisons différentes polices de caractère : Avenir pour Liria, et Goudy pour Paloma.

\section{Les débuts d'une amitié}

J'aimerais beaucoup qu'en lisant ce que je m'apprête à écrire, les gens comprennent à quel point ma rencontre avec mon amie Paloma fut formidable et importante pour moi. Je n'écrirai jamais assez pour exprimer ma gratitude envers une amie unique. Tout a commencé par une visite sur le terrain. Nous n'aurions jamais imaginé que cela aurait de telles répercussions sur nos vies. Nos vies commençaient à peine, nous avions toutes deux vingt ans, elle était célibataire et moi, mariée avec deux enfants, Nena et Angel. Même si nous avions grandi dans des environnements très différents, que j'étais gitane et elle paya, et que nous appartenions donc à des etnias, ethnies, différentes, nous avions tant de choses en commun. Cela ne nous a jamais éloignées l'une de l'autre, bien au contraire. Je pense même que c'est ce qui rend notre amitié intéressante, ce désir de connaître de nouveaux univers et de nouvelles personnes, différentes de celles avec lesquelles nous avions l'habitude de vivre.

C'est pour cette raison que je me souviens parfaitement du jour où j'ai rencontré Paloma. Ma sœur aînée Carmen m'avait déjà parlé d'elle. Elle m'avait raconté qu'elle avait rencontré une jeune fille paya qui fréquentait l'église de Villaverde et menait une étude sur les Gitans évangéliques, sur notre environnement et tout ce qui touchait aux Gitans du quartier. Lorsque ma sœur me l'a présentée, j'ai trouvé qu'elle avait un air ignorant et timide, mais je reconnais maintenant que c'était nous les ignorants, et qu'elle était en fait très courageuse d'être venue dans un quartier où il n'y avait presque que des Gitans, pauvres et peu éduqués pour la plupart. C'est pourquoi j'admets que son travail était difficile. Elle avait commencé par la partie la plus ardue, et il lui restait beaucoup de chemin à parcourir.

Ma première impression fut qu'elle était quelqu'un d'intelligent et plutôt sérieuse. Après nous avoir présentées, ma sœur m'avait précisé que Paloma cherchait à habiter avec une famille du quartier, mais que personne ne se proposait et que ses études en dépendaient. Je connaissais à peine Paloma, je l'avais seulement aperçue à l'église, je ne lui avais jamais parlé, mais ma sœur avait dit de très bonnes choses à son sujet, et m'avait expliqué qu'elle et son mari ne pouvaient pas l'accueillir car ce 
dernier était un pasteur évangélique. À moi aussi, on m'a conseillé de ne pas accueillir une fille paya chez moi parce qu'elle créerait des problèmes dans mon couple. Cependant, mon mariage ne pouvait pas aller davantage à vau-l'eau, même si à cette époque, mon mari n'était pas si mauvais avec moi. J'avais de la peine pour cette fille qui s'intéressait tant à nos vies et à notre mode de vie, et à qui nous ne donnions pas la chance de mener à bien son projet et son avenir. Mes parents ont alors appuyé ma décision d'accueillir Paloma chez moi. Ils ont toujours été très libéraux, en particulier ma mère, qui avait des amis de toutes les ethnies, et qui ne faisait aucun cas de la race, de la couleur ou de la situation sociale. J'admets également que j'avais moi aussi envie d'en apprendre davantage sur son monde. En effet, mes premières amies, quand j'étais petite, étaient des Payas qui allaient à l'école avec moi et j'aimais beaucoup leur façon d'être, si simple. Les Payos mènent en effet une vie plus indépendante et ne pensent pas à ce que pensent les autres ou aux ragots qu'ils peuvent exprimer. Cela m'a toujours dérangée de devoir faire des choses pour que les autres me laissent tranquille et ne me critiquent pas pour un oui ou pour un non. C'est pourquoi je souhaitais vivre une amitié avec une personne payo, d'autant plus que depuis mon mariage, tous mes amis étaient des Gitans.

Ainsi, en suivant mon cour et mon instinct, j'ai accepté qu'elle vienne vivre avec nous dans ma maison et qu'elle finisse ses recherches. D'une certaine manière, je l'étudiais moi aussi car j'étais fascinée par son monde et son mode de vie, même si je n'avais aucune idée de ce que la famille de Paloma pensait de nous, les Gitans.

Notre amitié est née de la bonté de Liria, qui m'a accueillie chez elle alors qu'elle me connaissait à peine et que ma présence n'augurait rien de bon. J'étais une Paya, jeune, sans attaches, et je ne parvenais pas vraiment à me faire accepter dans une communauté fortement marginalisée au sein de laquelle on se méfiait et on méprisait les Payos dominants et où les femmes payas étaient considérées immorales, avec des mœurs sexuelles légères. Liria est allée au-delà des stéréotypes et des conventions régissant les interactions entre Payos et Gitans, elle a remis en question ce que la plupart des personnes de son entourage prenaient pour acquis, et c'est uniquement pour cela que nous sommes devenues amies. Sa générosité, sa compassion et sa curiosité furent à la base de notre amitié.

Nous étions fascinées l'une par l'autre, peut-être parce que ni l'une ni l'autre n'étions satisfaites de nos vies et parce que nous incarnions l'une pour l'autre le profond désir inassouvi d'appartenir à un autre monde. J'avais grandi dans une famille conservatrice de la classe moyenne 
supérieure. J'avais appris plusieurs langues et voyagé régulièrement à l'étranger, mais j'avais aussi été plongée dans un monde aux convenances rigides dues à la classe sociale, à l'éducation, à la profession, à la façon de s'habiller ou à l'accent. Je me suis tournée vers l'anthropologie pour me réfugier dans des mondes imaginaires et alternatifs, mais je n'ai réussi qu'à troquer l'atmosphère suffocante d'une classe moyenne supérieure de Madrid repliée sur elle-même pour l'atmosphère suffocante d'une université de Cambridge. Je n'étais dans mon élément ni dans l'une ni dans l'autre. À Villaverde, parmi les Gitans, j'étais encore moins à ma place. En effet, lorsque j'ai rencontré Liria, cela faisait déjà neuf mois que je menais mes recherches sur le terrain et ma frustration augmentait. J'étais persuadée que je n'arriverais jamais à « m'intégrer ».

À première vue, Liria m'a semblé sûre d'elle et du chemin à prendre dans la vie. C'était une jeune matrone respectée, une bonne vendeuse de rue qui gagnait bien sa vie et suivait avec rigueur le très complexe code de conduite des Gitans destiné aux femmes. Elle portait toujours de modestes jupes longues, et ne fumait ni ne buvait jamais ou n'adressait jamais la parole à des hommes qui ne lui étaient pas apparentés. Ses parents étaient aisés par rapport à d'autres familles gitanes des environs et ils étaient très appréciés. L'importante parentèle du côté de son père était puissante et régissait la plus grande partie de la vie des Gitans du ghetto. Sa mère arrangea ses fiançailles à quinze ans avec un proche plus âgé, Ramón, qu'elle épousa lors d'une cérémonie de mariage de prestige au cours de laquelle sa virginité fut testée et affichée, plutôt qu'après la nuit de noces, comme ce fut le cas pour ses cousines et ses amies. Elle était intégrée, et pourtant, j'ai rapidement compris qu'elle n'était pas satisfaite, mariée à un homme qu'elle n'aimait pas et qui ne pouvait l'aimer, prise dans la routine quotidienne de l'épouse, et dans les restrictions imposées par son statut de una gitana decente, une Gitane respectable. Mais par dessus tout, elle cherchait désespérément à savoir comment se passaient les choses chez les Payos, qui l'entouraient mais demeuraient inaccessibles pour elle. Elle comprenait de manière intuitive mais profonde ce qu'était l'anthropologie et endossa le rôle 
d'informatrice avec enthousiasme.

Liria voulait en apprendre davantage sur les Payos et par conséquent, sur moi et ce qu'elle appelait tu mundo, ton monde. Ensemble, nous avons pris d'énormes risques, nous avons menti à Ramón et sommes sorties en secret à Madrid pour qu'elle voie ce qu'était ma vie. Nous nous habillions dans le style payo, nous débarrassant de nos longues jupes pour mettre des pantalons, que les Gitanes du ghetto ne portaient jamais à l'époque. Nous visitions des musées, allions dans des parcs, des restaurants pour la classe moyenne, et dans la maison où j'ai grandi. Comme elle m'avait ouvert sa demeure et sa vie, qu'elle était si curieuse de découvrir les miennes, j'estimais que je devais lui rendre la pareille et j'amenais Liria chez ma mère, dans son appartement, où elle rencontra ma famille mais également les domestiques qui travaillaient pour nous, puis à l'université, où nous dînions avec mes amis d'enfance, des garçons et des filles, étudiants en commerce, en droit et en économie. Tout comme les études sur le terrain parmi les Gitans l'étaient pour moi, ces escapades dans le Madrid de la classe moyenne avaient un parfum d'aventure pour Liria. Elle avait passé toute sa vie à la périphérie de la ville, et découvrait littéralement un nouveau Madrid. De plus, à l'université, elle parlait pour la première fois en toute liberté à des hommes de son âge qui ne lui étaient pas apparentés.

Nous nous amusions beaucoup ensemble, nous avions des conversations qui n'en finissaient plus, pendant que nous tenions le stand au marché, que nous cuisinions, que nous nous occupions des enfants et le soir, pendant que Ramón regardait la télévision. Nous parlions des hommes et de sexe, de notre passé et de notre avenir, du fait d'être gitane ou paya, et d'anthropologie. Nous débattions pour savoir si, en tant que Paya, j'avais réellement plus de liberté qu'elle, et quel genre de libertés. Je lisais à Liria des extraits du classique de l'ethnographie gitane de Teresa San Román, écrit dans les années 1970, et nous parlions des tenants et des aboutissants du récit anthropologique sur la filiation patrilinéaire chez les Gitans. Je lui lisais aussi des notes prises sur le terrain et nous riions de choses que nous avions dites quelques jours ou semaines plus tôt. L'amitié de Liria était un merveilleux cadeau. 
A posteriori, je me rends compte que les inégalités matérielles qui existaient entre nous ne nous préoccupaient pas, alors que désormais, leur importance semble si flagrante et qu'elles m'inquiètent tant. J'étais évidemment consciente des hiérarchies à grande échelle et des inégalités qui encadraient la marginalité des Gitans, et de nos positions relatives au sein même de cette dernière. Cependant, au quotidien, dans le ghetto, j'étais une étrangère qui cherchait à s'intégrer. Bien sûr, mes parents étaient plus aisés et j'en avais bénéficié : j'ai eu une vie agréable et l'occasion d'étudier à l'étranger. Mais Liria venait d'une famille gitane qui jouissait d'un grand respect à Villaverde et elle savait parfaitement où elle se situait au sein de la communauté gitane, dans laquelle les hiérarchies et les inégalités qui comptaient existaient parmi les Gitans, et où les Payos étaient des étrangers méprisés. À Villaverde, Liria avait sa place et un statut, mais moi, je n'avais aucun des deux. De même, il ne m'est jamais venu à l'esprit qu'en ouvrant ma vie à Liria, j'agissais peutêtre de manière contraire à l'éthique. Plus tard, alors que je parlais de notre amitié à un public anthropologique au Royaume-Uni, on m'a critiquée de ne pas avoir pris en compte l'impact que pourrait avoir sur Liria la rencontre avec ma famille, de ne pas avoir pensé qu'après cela, elle pourrait ne plus se satisfaire de son propre sort en tant que femme

gitane pauvre. À l'époque, nous savions toutes deux qu'il m'était impossible de demander à faire partie de la vie de Liria, sans la laisser approcher de la mienne.

\section{La phase intermédiaire}

Après avoir passé tellement de temps ensemble, des moments où elle venait chez moi pour son travail de terrain jusqu'à maintenant, je n'aurais pas assez de place pour noter toutes les bonnes et les mauvaises expériences que nous avons vécues ensemble. Je peux désormais dire de tout mon cœur que la relation que Paloma et moi entretenons est semblable à une relation entre sœurs, les amies ne devant pas uniquement être présentes lorsque tout va bien, mais également lorsque les choses tournent mal.

Pour moi, tout tournait autour du entorno, de l'environnement gitan, et lorsque Paloma vivait avec moi, le simple fait de la voir m'a ouvert les yeux. J'ai compris 
qu'une femme n'était pas seulement bonne à se marier, à avoir des enfants et à faire le ménage, même si chez les Gitans, je sortais régulièrement avec mes sœurs, nous allions à la plage l'été et en hiver, nous allions dans les centres commerciaux pour faire du shopping. Avec Paloma, je faisais des choses différentes. Nous allions au musée ou à l'université et faisions de nombreuses autres activités qui me plaisaient. Mais surtout, elle m'a amenée à voir mes qualités de femme. Elle me disait sans cesse que j'étais intelligente et que j'étais une bonne personne, alors que dans ma famille, on me traitait toujours comme une sorte d'idiote qui se faisait régulièrement mener en bateau. Paloma fut l'une des personnes qui m'ont fait prendre conscience de mes qualités et de ma valeur, en particulier par rapport à mon mari, Ramón. II savait comment m'embrouiller l'esprit en me laissant croire que je n'étais pas assez intelligente, ou jolie. Il me le répétait tant que j'ai fini par le croire. Jusqu'à ce qu'une formidable amie arrive et me dise que ce n'était pas vrai. Au fil des années, d'autres amies paya avec qui j'ai travaillé avec plaisir lorsque j'étais présidente de l'association des parents de l'école de ma fille m'ont également encouragée.

Entre 1993, fin de mes études sur le terrain dans le cadre de mon doctorat et 2008, Liria et moi sommes restées en contact. Nous nous écrivions régulièrement, nous téléphonions souvent et nous rencontrions à chaque fois que j'allais à Madrid pour voir ma mère et mes sœurs ou pour faire des recherches, c'est-à-dire au moins une fois par an. Avec le temps, nous partagions toujours nos joies et nos préoccupations par rapport à notre mari, notre travail, nos grossesses, nos enfants, l'école et notre famille. Pendant tout ce temps, j'ai écrit un livre et plusieurs articles à propos de Liria et de ses proches et voisins. Nous étions une anthropologue et son informatrice mais nous étions aussi des amies. Et c'est toujours en tant qu'amie que Liria m'aidait dans mon travail. Elle savait parfaitement ce qui m'intéressait : la vie des femmes, l'inégalité entre les sexes, la mort, l'église évangélique. Cependant, nous ne parlions jamais de ce que je faisais avec ce que j'apprenais, à qui je communiquais mes informations et la manière dont je le faisais, à qui celles-ci bénéficiaient ou de quelle manière. Je ne pouvais donner à Liria et aux autres Gitans qu'une vague idée de ce qu'impliquait mon travail, à savoir « enseigner » et « écrire », comme j'avais l'habitude de dire, et de la manière dont sont formés, traités et publiés les textes anthropologiques. Il me semblait presque impossible de transmettre à Liria les perspectives et théories par le biais desquelles sa vie pouvait être significative d'un point de vue anthropologique. Et comme je 
travaillais au Royaume-Uni et que j'avais choisi d'écrire en anglais pour un auditoire universitaire, Liria ne pouvait même pas lire le fruit de mon travail.

Pendant cette période, le mariage de Liria est allé de mal en pis, et elle a quitté Ramón à plusieurs reprises pour s'installer chez ses parents avec ses enfants. Sa famille la persuada à chaque fois de retourner auprès de son mari. En 2008, elle devient présidente de l'association de parents de l'école de sa fille et milite brièvement contre la ségrégation des enfants gitans dans les écoles situées à proximité du ghetto. Ma mère m'appelait, tout excitée, lorsqu'elle l'apercevait au bulletin d'informations de midi. Au même moment, je prenais moi aussi un nouveau chemin. J'ai eu un fils, puis j'ai adopté une petite fille en Chine. Je me suis davantage engagée sur la scène politique, j'ai commencé à travailler à temps partiel et ma carrière a pris la deuxième, voire la troisième place dans ma vie. Nos horizons à toutes deux se sont éclaircis pendant les années 2000. Pour moi, au-delà du monde de l'anthropologie et du milieu universitaire et pour Liria, au-delà de sa famille et de l'église évangélique gitane. Puis, Liria a rencontré Younes par hasard, et nos vies nous ont rapprochées plus encore qu'auparavant.

\section{Des vies transformées}

Un matin comme tant d'autres, l'inattendu se produisit. Un jeune homme travaillait avec des amis à moi sur un étal non loin du mien, seuls quelques marchands de fruits nous séparaient. Je ne sais pas pourquoi un matin, je me suis retrouvée devant l'étal de mes amis pour les saluer et voir les vêtements qu'ils vendaient. Ils avaient souvent de beaux articles et j'aimais leur en acheter. En vérité, j'avais déjà remarqué ce garçon, mais la honte et la peur de tomber amoureuse, en particulier d'un homme plus jeune que moi, m'interdisaient de faire attention à lui ou à n'importe qui d'autre. Mais ce matin-là, pour je ne sais quelle raison, je le regardai et son regard plongea profondément dans le mien. J'avais l'impression que ses yeux me parlaient, je n'avais jamais rien ressenti de tel.

Les sœurs de Liria m'appelèrent de Madrid. Elle avait disparu la veille et elles étaient désespérées. Elles avaient trouvé le nom d'un homme et son 
numéro de téléphone inscrits sur un morceau de papier dans un des sacs à main de Liria, et la soupçonnaient de s'être enfuie avec lui. J'étais censée les prévenir sans attendre si Liria me contactait. J'ai essayé à maintes reprises de joindre Liria par téléphone et par texto : «Où es-tu? Tout le monde est inquiet. Est-ce que tout va bien? Appelle-moi STP, je meurs d'angoisse. » Elle m'appela ce soir-là. Elle était partie avec Younes, un jeune homme qu'elle avait rencontré tout récemment. Ses sœurs avaient découvert qu'elle avait une liaison et par crainte de la réaction de sa famille, elle avait estimé que sa seule option était de fuir sur-le-champ. De plus, elle avait essayé de vivre avec Ramón pendant 20 ans et Younes l'aimait. Elle n'avait pas réussi à emmener sa plus jeune fille. En effet, selon le droit coutumier des Gitans, souvent appliqué par la force, en cas d'adultère, les enfants doivent rester avec celui ou celle qui n'a rien à se reprocher. Ses sœurs et beaux-frères étaient donc à sa recherche pour la ramener et peut-être les punir, elle et Younes. Elle était terrifiée. J'étais censée dire qu'elle ne m'avait pas contactée et garder son secret afin qu'elle soit en sécurité.

Mes sœurs et leurs maris ont fini par me retrouver et je n'eus pas d'autre choix que de rentrer car ma famille menaçait de tuer Younes. Aujourd'hui, je me rends compte que je me suis laissé intimider et que la peur m'a empêchée de réfléchir clairement. Je comprends désormais qu'ils auraient pu faire du mal à Younes avant de venir me chercher dans l'appartement où je m'étais cachée. Ils se sont retrouvés seuls avec lui en bas pendant un long moment, mais ils ne lui ont rien fait. En fait, ils m'ont convaincue en usant de menaces et de gentillesse à la fois, en faisant tout ce qui était en leur pouvoir, car ils étaient désespérés à ce moment-là. Cela fut très douloureux pour moi. D'abord, il y avait Younes et le fait de devoir le quitter. Je ne savais pas comment lui expliquer que ma famille craignait qu'il ne m'ait dupée ou forcée à le suivre, car c'était la première fois que je faisais quelque chose comme ça. Et puis, il y avait mes enfants. Lorsque je suis rentrée, j'ai eu le cœur brisé en voyant à quel point je leur avais manqué. "Comment vais-je pouvoir récupérer ma famille et mes enfants ? " Voilà ce à quoi je pensais à ce moment-là. Mais il était trop tard, plus personne ne me faisait confiance, j'étais surveillée de près tout le temps.

Liria a dû rentrer et lors de ma visite à Madrid, trois semaines plus tard, toute sa famille voulut s'assurer que je comprenais pourquoi ce qu'elle avait fait était mal. «C'est comme ça que nous, les Gitans, faisons les choses, tu nous connais, tu nous comprends, tu sais à quel point ceci est 
terrible pour nous. Nous ne sommes pas comme vous, les Payos, cela est totalement inacceptable pour nous, c'est le pire qui puisse arriver. » Ils disaient que je devais convaincre Liria de ne plus s'enfuir, aider à faire en sorte qu'elle reste à la maison, sous leur contrôle. Ramón, Carmen et les autres sœurs de Liria, ses enfants, sa belle-fille, ils incarnaient tous la voix de la raison des Gitans. Ils m'avaient soutenue dans mon travail et savaient comme Liria et moi étions proches; ils tenaient absolument à ce que je prenne parti. Ils étaient « les Gitans » à propos desquels j'avais écrit pendant de nombreuses années et ce qu'ils disaient correspondait en tous points à ce que j'avais appris à leur sujet : la vertu et la soumission des femmes face aux hommes sont essentielles dans la manière dont elles voient leur place dans le monde. Cependant, elle m'appelait à l'aide. Il était question ici de Liria, mon amie, une femme dont je connaissais les craintes et les désirs, qui m'avait fait part de ses souhaits et de ses déceptions, qui m'aimait et que j'aimais. Je ne lui ai donc pas dit de « partir » ou de « rester », mais je l'ai aidée à voir Younes en cachette. Pour nous couvrir, nous emmenions nos plus jeunes enfants. Absorbés par un nouveau jeu Nintendo, ils n'avaient aucune idée de ce qui se passait autour d'eux, et nous savions que la famille ne nous croirait jamais capables d'une telle audace. Lorsque Liria décida de partir pour de bon, je l'aidai à nouveau, élaborant des plans, l'écoutant exprimer ses craintes, son anxiété et ses espoirs et je leur fournis une partie de l'argent dont ils avaient besoin pour tout recommencer. À partir du moment où Younes et elle se sont cachés, je suis devenue le seul contact entre Liria et sa famille, lui passant les plaintes de ses enfants qui me brisaient le cœur, recevant et relayant les lettres de désespoir, de supplication et de colère de Ramón.

J'ai le cour brisé. Chaque jour qui passe, je me sens encore plus mal, pour ma fille. À chaque fois que je vois des filles de son âge dans la rue, je meurs à l'intérieur, vraiment. Quelque chose me tue à l'intérieur. J'essaie de ne pas en parler à Younes et je vais dans la salle de bain pour pleurer. Je me dis « Sois heureuse ». Mais comment puis-je être heureuse quand je sais que ma fille a besoin de moi ? Puis je me répète : «Et si j’y retourne et que je meurs à force de me languir de Younes ? » Je ne pense qu'à elle, je n'ai que ça en tête.

Pendant six mois, Liria vécut avec Younes, dans des appartements qu'ils 
partageaient avec des immigrés venus d'Afrique et d'Amérique Latine. Elle travailla comme domestique, tentant de cacher son identité gitane à ses employeurs, des gens de la classe moyenne, pas très différents de ma propre famille. Nous nous parlions presque tous les jours et je lui rendais visite toutes les deux semaines. Je voyais bien comme Younes et elle s'aimaient, tout le plaisir et la liberté dont elle jouissait dans sa nouvelle vie, mais je mesurais également l'ampleur de son désespoir. Je la voyais pleurer lorsqu'elle tenait ma fille dans ses bras, en proie aux remords. J'étais en colère contre Ramón et les sœurs de Liria qui demeuraient inflexibles : elle ne verrait pas son enfant tant qu'elle ne reviendrait pas. Et si elle prenait l'enfant, me disaient-ils, ils les tueraient, elle et Younes. Je comprenais bien la logique proprement culturelle et la profonde douleur qui dictaient leurs actions et je savais que je ne devais pas m'attendre à un comportement différent ; cependant, je m'interrogeais. Je me suis mise à me questionner quant à la puissance de la compassion et aussi de la haine. Ramón et les sœurs de Liria ne pouvaient-ils pas la prendre en pitié, simplement parce qu'ils étaient Gitans ? Étaient-ils si stricts parce qu'ils étaient Gitans ou parce qu'ils souffraient? Nous ne voyions pas comment faire évoluer la situation et une fois encore, Liria finit par rentrer.

\section{Écrire ensemble}

En 2009, la première fois que Liria est partie de chez elle, mais aussi plus tard, Younes et elle avait grandement besoin d'argent. Ils devaient se cacher afin que la famille étendue de Liria ne les retrouve pas, et ils perdirent leur gagne-pain. Liria ne pouvait plus vendre au côté de Ramón et Younes ne pouvait plus travailler sur les marchés à décharger les biens pour les vendeurs gitans, comme il le faisait depuis son arrivée à Madrid. La crise économique s'intensifiait et il était désormais presque impossible de trouver du travail. Sans papiers, Younes ne pouvait trouver que des emplois de courte durée et très mal payés.

Je savais qu'il me serait difficile de fournir une aide économique substantielle à Liria et Younes pendant une période prolongée. J'ai donc postulé tout d'abord auprès de mon département au Royaume-Uni, puis 
j'ai demandé une faible subvention afin de pouvoir payer Liria pendant qu'elle rédigeait son autobiographie. Liria s'est donc mise au travail, elle a fini par produire quarante pages manuscrites traitant de ses expériences lors de la protestation contre la ségrégation des enfants gitans dans l'école de son quartier et de son départ de la communauté gitane. À ce moment, j'effectuais des recherches sur l'adoption internationale et je pensais que mon travail sur les Gitans était terminé. Mais nous nous sommes très rapidement rendu compte que nous avions une histoire très importante à raconter. Ce qui avait donc commencé comme une manière de trouver de l'argent est devenu un projet fascinant pour nous deux. Nous avons décidé de travailler ensemble sur l'histoire d'une vie.

Je n'étais pas qu'une observatrice dans la vie de Liria, j'y jouais un rôleclé. Nous savions que le livre raconterait son histoire, mais également mon rôle dans celle-ci et la place que l'anthropologie occupait dans notre amitié. De plus, étant donné que Liria avait commencé à écrire, il était clair dès le début que nous serions co-autrices. Plus tard, le projet a changé. Nous avons pris conscience que pour mieux transmettre la nature de notre relation, et les expériences vécues par Liria en tant que femme gitane, nous devions mettre par écrit ce que nous avions fait oralement pendant de nombreuses années : nous pencher ensemble sur nos vies respectives, en tant que Paya et Gitane, chacune analysant l'autre, soi-même et le monde qui nous entoure.

Écrire s'avérait laborieux pour Liria, elle s'est donc mise à enregistrer ses réflexions. Je m'occupais de transcrire ces enregistrements et les textes de Liria en y ajoutant la ponctuation tout en essayant de conserver le plus possible le ton et le style qu'elle employait. Je me suis également mise à écrire, toujours en anglais pour commencer, avant de traduire ce que j'écrivais pour Liria. Étant donné que j'ai étudié et enseigné au Royaume-Uni, il est beaucoup plus naturel pour moi de rédiger mes travaux d'anthropologie en anglais. Ensemble, nous avons parcouru les lettres que Liria m'avait envoyées au cours des vingt dernières années, mais malheureusement, elle avait perdu toutes celles que je lui avais envoyées au moment de s'enfuir de Villaverde. 
Dans notre projet, la limite entre travail de terrain et écriture n'est pas très claire. Notre collaboration remet en cause la séparation entre le terrain et le bureau, et entre le sujet et l'ethnographe sur laquelle repose l'anthropologie en tant que matière académique. Dans le même temps, le projet est basé sur nos différentes positions et contributions, et même sur les inégalités qui existent entre nous. Comme ces différences représentent la source de notre force commune, nous les laissons clairement apparaître dans nos écrits. Nos voix s'expriment à l'unisson afin que nos deux formes de savoir et nos manières différentes de nous investir dans l'anthropologie et dans le monde bénéficient de la même visibilité et de la même pertinence.

Alors que ma formation en anthropologie me pousse à trier, analyser, établir une structure et une argumentation, et que j'ai tendance à me tourner vers les contextes sociaux et culturels de la vie des gens, Liria écrit de manière beaucoup plus fluide et spontanée. Elle recherche davantage les motivations internes de nos actions et de celles des autres. Nos espoirs et objectifs quant à ce projet sont aussi différents. Pour Liria, ce travail se veut un témoignage qui lui permet de dire aux autres ce qu'être être une femme gitane signifie. Elle croit fermement que notre relation, en tant que Paya et Gitana, hermanas del alma, âmes-sœurs, est un exemple à suivre pour les autres. Elle est une fervente croyante de l'Église évangélique, persuadée que Dieu parlera à nos lecteurs à travers ce livre. Pour moi, le projet représente une chance de grandir et d'évoluer en tant qu' anthropologue, en mettant à l'essai un style d'ethnographie ouvert et réciproque.

L'anthropologie est à l'origine de notre relation et continue à la modeler et à l'enrichir. Par conséquent, nous cherchons à faire ressortir les effets de l'anthropologie sur nos vies. Le travail de terrain est souvent décrit comme le parcours des découvertes faites par un ethnographe, mais notre histoire démontre également l'importance de l'anthropologie pour ceux et celles qui demeurent sur le terrain lorsque débute la phase d'écriture. Comme le dit Liria, depuis notre première rencontre, l'anthropologie a été pour elle une manière de comprendre le monde et de se comprendre elle-même, tout à fait comme cela fut le cas pour moi. 
De même, alors que l'écriture ethnographique est le plus souvent vue comme un processus par lequel un anthropologue devient auteur et trouve un point de vue original sur le monde, nous soulignons que la production de connaissance ethnographique est également un processus au cours duquel les informateurs peuvent être aussi actifs que l'ethnographe, même s'ils sont plus discrets.

Il s'agit là d'une collaboration entre deux individus, plutôt qu'entre une communauté ou un groupe et une anthropologue : de nombreuses voix sont mises de côté. En écrivant un ouvrage anthropologique plutôt que de produire un document que l'auditoire composé de ces Gitans, de ces Payos et de ces immigrants pourrait trouver utile, pertinent ou intéressant tel qu'un documentaire, une biographie populaire ou un ouvrage de photographies, nous les avons peut-être aussi exclus.

Le fait d'analyser nos histoires en parallèle nous permet de nous interroger sur les contraintes et les possibilités qui existent dans la vie des femmes, Gitanes et Payas, dans l'Espagne contemporaine, et sur les cadres de la hiérarchie et de l'inégalité qui les régissent. Enfin, notre approche biographique, réflexive et intime, nous permet d'aborder des thèmes que les anthropologues qui écrivent à propos des Tsiganes ou Roms ont jusqu'à présent trouvé difficiles à traiter : pas simplement l'individualité et la situation mais d'une manière cruciale, les liens profonds qui entrelacent la vie des Tsiganes ou Roms et de celles et ceux qui ne le sont pas. 